\title{
Crown-Ether End-Capped Carbosiloxane Dendrimers
}

\author{
Chungkyun Kim and Hyojeong Kim \\ Department of Chemistr, Dong-A Lniversity, Busan 604-714, Korea \\ Recerved Januarv 2, 2002
}

Keywords : Dendrimer. Crown ether. Carbosilane. Hydrosilation Siloxane.

Dendrimers are perfect and unified macromolecules with regular and highly branched architectures that are obtained from an iterative procedure. 'Due to their chemical and physical properties. dendrimers are used for many fields in material science. The end-functionalized dendritic macromolecules with specific groups were studied by many researchers who used the electro-active groups or molecular recognition groups that could find applications as components in sensors and electro-devices etc. ${ }^{3}$ Especially, crownether end-capped dendrimer and polymer could possibly be used as the high ion selective sensor. ${ }^{-c} \ln$ our previous paper. we described the preparation of ally loxy and propagyloxy group functionalized carbosiloxane dendrimers. based on siloxane tetramer $\left(\mathrm{MeSiOCH}=\mathrm{CH}_{2}\right)_{4}$ as a core molecule and methylallyloxy groups as generating units. ${ }^{4}$ For the purpose of extending of applicability: we have shifted our attention to the preparation of crown-ether end-capped dendrimers. ${ }^{5}$

The parent dendrimers. constructed with $\mathrm{Si}-\mathrm{Cl}$ bonds on the fourth and fifth generations. were prepared by the use of catalytic hyddrosilation with allyloxy groups on the dendrimers and $\mathrm{H}-\mathrm{SiMe}_{2} \mathrm{Cl}^{5}$ "The reaction of the 4 th $(\mathrm{G} 4-48 \mathrm{Cl})$ and 5 th $(\mathrm{G5}-96 \mathrm{Cl})$ generations of the parent dendrimers with crown-ether (2-(hydroxymethyl)-12-crown-4 and 2-(hydroxymethyl)-15-crown-5) in the presence of TMEDA produced crown-ether end-capped dendrimers with a very high yield (Scheme 1). The Si-O-C bonds on the outmost periphery are very stable against air and moisture. Therefore. after alcoholysis with 2-(hyddroxymethyl)-12-crown-4 and 2-(hydroxymethyl)-15-crown-5. the handling of the prepared dendrimers in the atmosphere is possible. The successful addition of crown-ether to parent dendrimers can be easily detected by the characteristic shift of the dimethylsilyl group in the ${ }^{13} \mathrm{C}$. NMR spectra. A shift of the ${ }^{13} \mathrm{C}$ NMR signals ranges from $3.50 \mathrm{ppm}$ for the dimetlylsilyl groups of the parent dendrimers $(\mathbf{G}+\mathbf{- 4 8 C l}$ and $\mathbf{G 5}-\mathbf{9 6 C l})$ to $0.99 \mathrm{ppm}$ in the case of G4-48Crown-4 or to $-2.22 \mathrm{ppm}$ in the case of G596Crown-4. Both dendrimers can be observed in oxo-ether groups on crown-ether ring at 62.6.70.2. 70.5, 70.6.70.9 and $71.4 \mathrm{ppm}$. The G4-48Crown-5 and G5-96Crown-5 revealed the same evidence at $-2.18 \mathrm{ppm}$ for both dendritic dimethylsilyl groups and oxo-ether groups at $62.6,70.3$. 70.5. 70.7, 71.0. and 71.1 (Figure 1). The G+48Crown-4. G5-96Crown-4, G4-48Crown-5 and G5-96Crown-5 could be prepared in 55,66, 53 and $60 \%$ yield after flash

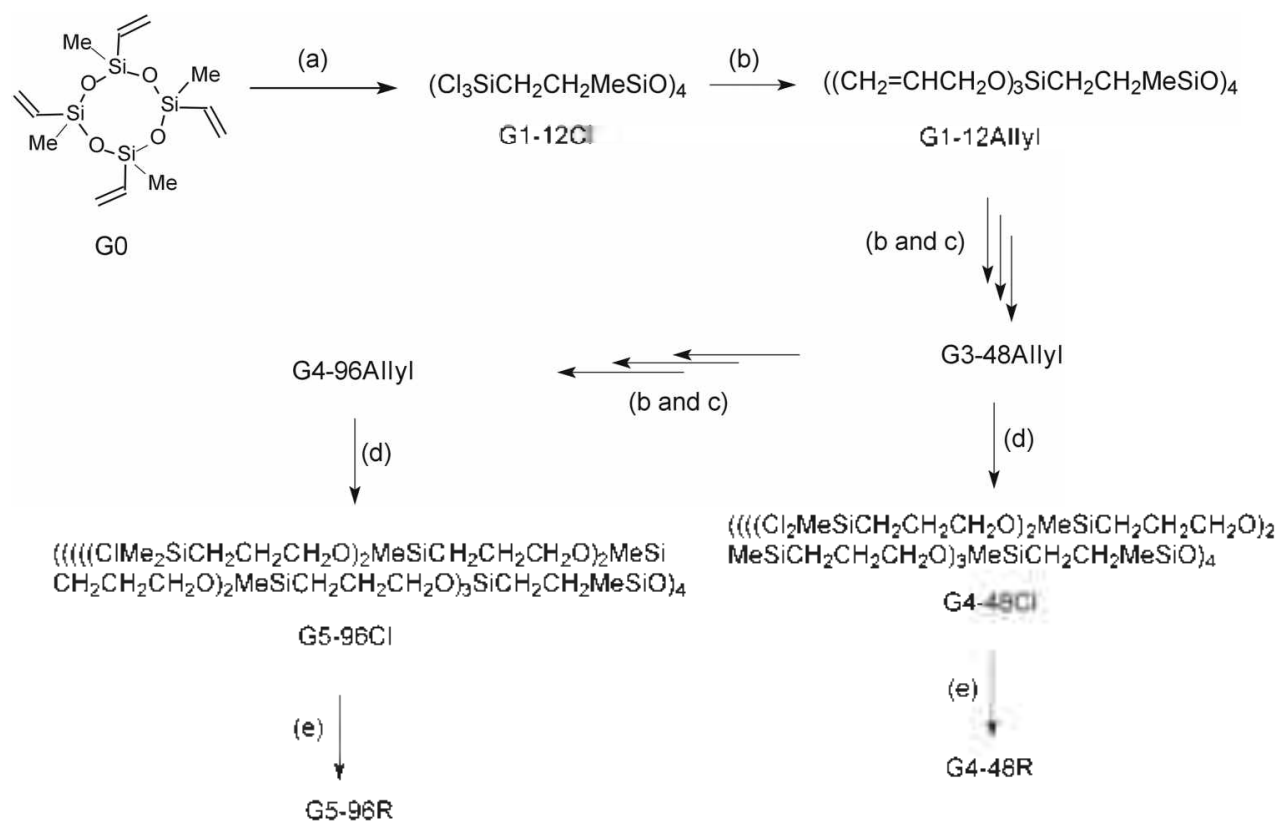

(a) Hydrosilation with $\mathrm{HSiCl}$. Pt/C, toluene, reflux (b) Alcoholysis with $\mathrm{CH}_{2}=\mathrm{CHCH}_{2} \mathrm{OH}_{1}$ TMEDA, RT $-50^{\circ} \mathrm{C}$, toluene
(c) Hydrosilation with $\mathrm{HSiMeCl}$ (d) Hydrosilation on the terminal groups with $\mathrm{HSiMe}{ }_{2} \mathrm{Cl}$
(e) Temnination with crown ether; $\mathrm{R}=2$-Methoxy-12-crown-4 and 2-Methoxy-15-crown-5, excess TMEDA, RT. toluene.

Scheme 1. Overview of reaction toute of crown-ether end capped dendrimers. 


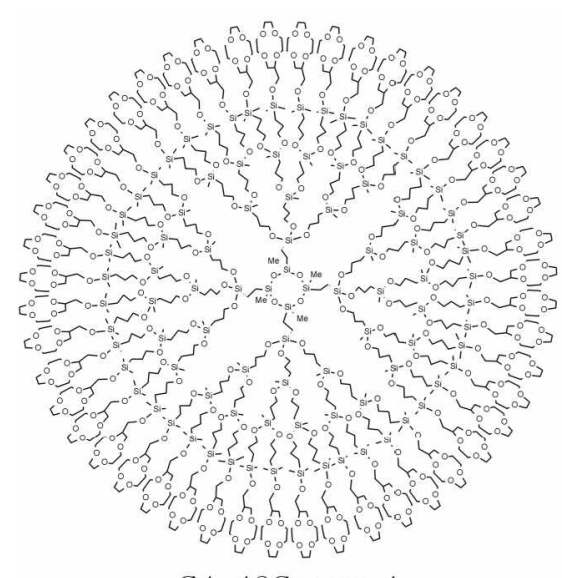

G4-48Crown-4

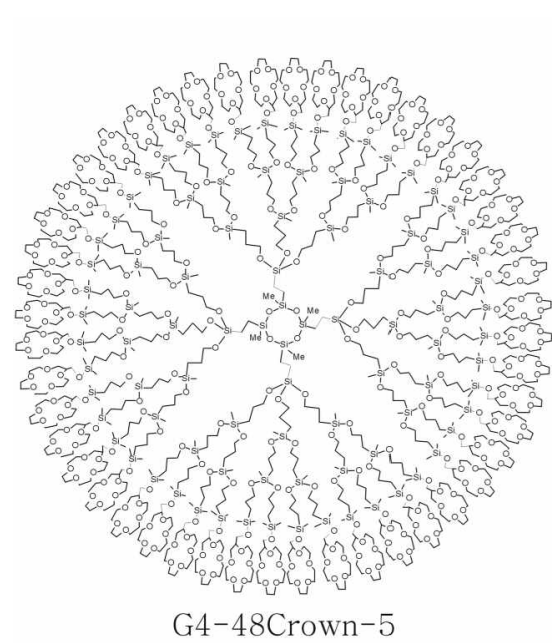

Figure $1 .{ }^{13} \mathrm{C}$ NMR spectra of crown ether end-capped dendrimers.

chromatography. respectively. The isolation of pure dendrimers from the reaction mixture was progressed under flash chromatography with chloroform as well as mixed eluents such as chloroform. THF $(9 ; 1)$ and silica gel columns. The identification of the end-capped dendrimers was done by the NMR, GPC as well as elemental analysis. The crown ether end-capped dendrimers could not provide MALDI-TOF-MS signals. The polydispersity index ( $\mathrm{PDl}$ ) values on gel permeation chromatography (GPC) remained almost unchanged in going from the fourth to the fifth generation $(1.03-1.04)$. Therefore. the end-capped dendritic macromolecules with fourth and fifth generations were grossly estimated to structural perfection.

\section{Experimental Section}

All reactions were carried out under dried $\mathrm{N}_{2}$ atmosphere. NMR spectra were recorded on a Bruker AC-200 Spectrometer. Size exclusion chromatography was performed in $\mathrm{THF}$ at $25^{\circ} \mathrm{C}$ with a Waters $515 \mathrm{HPLC}$ pump together with a Waters 2410 Refractive Index Detector. Three $7.8 \times 30 \mathrm{~cm}$ colunns (Ultrastyragel) were connected in series, calibrated with narrow molecular weight polystyrene standard. Low

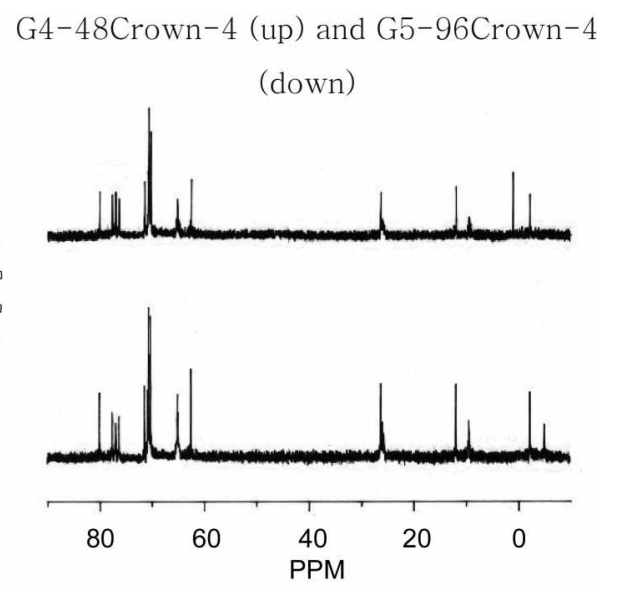

G4-48Crown-5 (up) and G5-96Crown-5

(down)

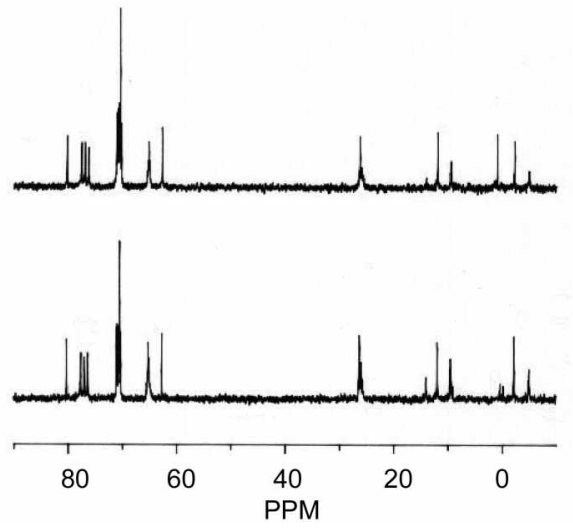

generational dendrimers (G1-G4) were prepared according to previous works.'

G4-48Crown-4 (Mw: 19.538). A mixture of 2-(hydrosymethyl)-12-crown-4 (0.43 g. $2.10 \mathrm{mmol}$ dissolved in $25 \mathrm{~mL}$ of THF) and $0.24 \mathrm{~g}$ of TMEDA was slowly added to G4$48 \mathrm{Cl}(0.47 \mathrm{~g} .0 .04 \mathrm{mmol})$ in $50 \mathrm{~mL}$ of toluene. After the addition was finished. the reaction mixture was warned up to $50^{\circ} \mathrm{C}$ for $\mathrm{l} \mathrm{h}$. Amine salt was filtered off. leaving $0.85 \mathrm{~g}$ of a light yellow solid. This was chromatographed on a silica gel with chloroform. Yield: $0.56 \mathrm{~g}(0.0 \mathrm{l} 4 \mathrm{mmol}, 55 \%)$ of a colorless gel. 'H NMR (ppm. CDCl ${ }_{2}$ ): $\delta=0.04$ (s. $120 \mathrm{H}$. SiMe. G0-G3). 0.09 (s. 288H. SiMe. G4) 0.47-0.76, 1.491.83 (m. 352H. CH. G0-G3). 3.40-3.90 (m. 984H, OCH G1-G3 and crown ether) ${ }^{13} \mathrm{C}$ NMR (ppm. $\left.\mathrm{CDCl}_{3}\right): \delta=-2.25$ (SiMe. G0-G3). 0.99 (SiMe. G4). 9.38 ( $\mathrm{CH}_{\beth} \mathrm{G} 0$ ). 11.87. $26.24\left(\mathrm{CH}_{2} . \mathrm{G} 3\right) .9 .38,26.24\left(\mathrm{CH}_{2}, \mathrm{Gl}-\mathrm{G} 2\right) .65 .20\left(\mathrm{OCH}_{2}\right.$. $\mathrm{Gl}-\mathrm{G} 3), 80.06\left(\mathrm{OCH}_{2}, \mathrm{G} 4\right), 62.67,70.26,70.54,70.64$. 70.79, 71.45 (OCH $\mathrm{H}_{2}$ and crown-ether). GPC: PDI $\left(\mathrm{M}_{w} / \mathrm{M}_{\mathrm{n}}\right)$. 1.05 (5082/4853): Rt. $16.05 \mathrm{~min}$. Anal. calcd. for $\mathrm{C}_{88} \mathrm{H}_{17+4} \mathrm{Si}_{92} \mathrm{O}_{3} 8:$ C. $50.89:$ H. 9.02\%. Found: C. 49.38: H. $9.36 \%$.

G5-96Crown-4 (Mw: 39.892). The same procedure as that for G4-48Crown-4 was used in the reaction of $0.60 \mathrm{~g}$ 
$(0.026 \mathrm{mumol})$ of $\mathrm{G} 5-96 \mathrm{Cl}, 0.54 \mathrm{~g}(2.63 \mathrm{mmmol})$ of 2 . (hydroxymethyl)-12-crown-t and $0.58 \mathrm{~g}(4.92 \mathrm{mmol})$ of TMEDA. The product was chromatographed on a silica gel with chloroform and a mixed eluent $\left(\mathrm{CHCl}_{\hat{j}}\right.$ : THF $\left.=9: 1\right)$. Yield: $0.36 \mathrm{~g}(0.016 \mathrm{~mm}$ l. $66 \%)$ of a colorless gel. ${ }^{1} \mathrm{H}$ NMR (ppm, CDCl $)_{3}$ ) $\delta=0.09$ (s. 26+H. SiMe. G0-G4). 0.43$0.77,1.45-1.75$ (m. $736 \mathrm{H}, \mathrm{CH}_{2}$. G0-G4), 3.40-3.94 (m, 1992 H, OCH . Gl-G4 and crown-ether) ${ }^{13} \mathrm{C}$ NMR (ppm, $\mathrm{CDCl}_{3}$ ): $\delta=-4.99$ (SiMe, G0-G4). -2.22 (SiMe, G5), 9.46 $\left(\mathrm{CH}_{2} . \mathrm{G} 0\right), 11.92 .26 .26\left(\mathrm{CH}_{2} \mathrm{G} 4\right), 9.40 .25 .99\left(\mathrm{CH}_{2}, \mathrm{Gl}-\right.$ G3). $65.18\left(\mathrm{OCH}_{2}, \mathrm{Gl}-\mathrm{G} 4\right), 80.11\left(\mathrm{OCH}_{2}, \mathrm{G} 5\right) .62 .54 .70 .30$, 70.61, 70.67. 70.86.71.47 $\left(\mathrm{OCH}_{2}\right.$ crowl-ether). GPC: PDI $\left(\mathrm{M}_{\mathrm{w}} / \mathrm{M}_{\mathrm{n}}\right), 1.05(6036 / 5766)$ : Rt, $15.88 \mathrm{~min}$. Antal. calcd. for $\mathrm{C}_{1693} \mathrm{H}_{3567} \mathrm{Si}_{188} \mathrm{O}_{664}:$ C. $51.02: \mathrm{H}, 9.05 \%$. Found: C. 49.99: $\mathrm{H}$. $8.76 \%$

G+-48Crown-5 (Mw: 21.651). The same procedure as that for G4-48Crown-4 was used in the reaction of $0.28 \mathrm{~g}$ $(0.026 \mathrm{mumol})$ of $\mathrm{G} 4-48 \mathrm{Cl}, 0.33 \mathrm{~g}(1.30 \mathrm{mmol})$ of 2 (hydroxymethyl)-15-crown-5 and $0.15 \mathrm{~g}(1.30 \mathrm{mmol})$ of TMEDA. The product was chromatographed on a silica gel with chloroform and a mixed eluent $\left(\mathrm{CHCl}_{3}: \mathrm{THF}=9: 1\right)$. Yield: $0.4 \mathrm{lg}(0.021 \mathrm{mmol} .53 \%)$ of a colorless gel. ${ }^{1} \mathrm{H}$ NMR (ppn. $\mathrm{CDCl}_{3}$ ): $\delta=0.09$ (s, 408H, SiMe. G0-G4). 0.45-0.73. 1.43-1.73 (m, 352H. $\mathrm{CH}_{2}, \mathrm{G} 0-\mathrm{G} 3$ ), 3.50-3.88 (m. $1176 \mathrm{H}$. $\mathrm{OCH}_{2} \cdot \mathrm{Gl}-\mathrm{G} 3$ and crown ether). ${ }^{13} \mathrm{C}$ NMR (ppm, $\mathrm{CDCl}_{3}$ ): $\delta$ $=-4.31$ (SiMe, G0-G3). -2.18 (SiMe. G4). 9.00 (CH. G0). $9.54,26.05\left(\mathrm{CH}_{2} . \mathrm{Gl} . \mathrm{G} 2\right) .12 .88,26.32\left(\mathrm{CH}_{2} . \mathrm{G} 3\right) .65 .24$ $\left(\mathrm{OCH}_{2} . \mathrm{Gl}-\mathrm{G} 3\right), 80.24\left(\mathrm{OCH}_{2} . \mathrm{G} 4\right) .62 .68,70.31 .70 .54$. $70.70,74.90 .71 .00 .71 .12\left(\mathrm{OCH}_{2}\right.$ crown-ether $)$ GPC: PDI $\left(\mathrm{M}_{\mathrm{W}} / \mathrm{M}_{\mathrm{n}}\right), 1.03$ (5733/5580): Rt, $15.98 \mathrm{~min}$. Anal. calcd. for $\mathrm{C}_{2} \mathrm{H}_{1936} \mathrm{Si}_{2} \mathrm{O}_{376}$. C. 51.25: H. 9.03\%. Found: C, 50.13; $\mathrm{H}$, $9.25 \%$.

G5-96Crown-5 (Mw, 44,059). The same procedure as that for G4-48Crown-4 was used in the reaction of $0.23 \mathrm{~g}$ $(0.01 \mathrm{~nm}$ ol $)$ of $\mathrm{G} 5.96 \mathrm{Cl}, 0.32 \mathrm{~g}(1.28 \mathrm{~nm}$ ol $)$ of 2 - (hydroxymethyl)-15-crompl-5 and $0.15 \mathrm{~g}(1.30 \mathrm{mmol})$ of TMEDA. The product was chromatographed on a silica gel with chloroform and a mixed eluent $\left(\mathrm{CHCl}_{3}: \mathrm{THF}=9: 1\right)$. Yield: $0.25 \mathrm{~g}(0.006 \mathrm{~mm}$ ol. $60 \%)$ of a colorless gel. ${ }^{1} \mathrm{H}$ NMR (ppm, $\mathrm{CDCl}_{3}$ ): $\delta=0.09$ (s, 264H. SiMe. G0-G4), 0.16 (s, 576H. SiMe. G5). 0.39-0.72, 1.41-1.76 (m, 736H, $\mathrm{CH}_{2}, \mathrm{GO}-$ G4). 3.52-3.84 (m. 2376H. OCH, Gl-G4 and croml-ether). ${ }^{13} \mathrm{C}$ NMR (ppm, $\mathrm{CDCl}_{3}$ ): $\delta=-4.97$ (SiMe. G0-G4). -2.18 (SiMe, G5). $9.00\left(\mathrm{CH}_{2}, \mathrm{G} 0\right)$. 11.48. $26.29\left(\mathrm{CH}_{2} . \mathrm{G} 4\right) .9 .48$, $26.26\left(\mathrm{CH}_{2} . \mathrm{Gl}-\mathrm{G} 3\right), 65.24\left(\mathrm{OCH}_{2} . \mathrm{Gl}-\mathrm{G} 5\right) .80 .24\left(\mathrm{OCH}_{2}\right.$, G5). 62.68, 70.31, 70.54. 70.70. 70.90, 71.00. 71.12(OCH crowll-ether). GPC: PDI $\left(\mathrm{M}_{w} / \mathrm{M}_{\mathrm{n}}\right), 1.06$ (7739/7311): Rt. $15.55 \mathrm{~min}$. Anal. calcd. for $\left(\mathrm{C}_{1884} \mathrm{H}_{395} \mathrm{Si}_{188} \mathrm{O}_{76 i}\right)$ : C, 51.36; H, 9.06\%. Found: C. 49.38; H, 8.43\%.

Acknowledgment. This study was supported by the Basic Research Program of the Korea Science \& Engineering Foundation (Grant No: R01 2000 0046).

\section{References}

1. (a) Vogtle. F: Gestermann. S.: Hesse, R:; Schwierz, H.; Windisch, B. Prog. Polv Sci. 2000. 25.987-1041. (b) Inoue. K. Prog. Polnt. Sci. 2000. 25. 453-571. (c) Newkomme. G. R.: He. E.: Moorefield. C. N. Chent Rev: 1999. 99. 168-1746. (d) Majoral. I.-P: Caminade. A.-M. Chem. Rev 1999, 99, 845-880. (e) Frey. H.; Shlenk. C. Top. Cwr. Chem 2000. 210, 69-129.

2. (a) Albrecht, M:; Gossage, R. A.; Speck, A. L.: van Koten. G. Chem. Commun. 1998. 1003-10044 (b) McElhation. J. R.: McGrath. D. V. J. Ant Chem Soc. 1998. 120. 1647-1656. (c) Chauhan1. B. P. S.: Boudjouk. P. Tetrahedron Lett. 1999. 40. 4123-4126.

3. (a) Reetz. M. T: Lohmer, G; Schwichardi, R. Angew: Chem. Int. Ed. Engl. 1997. 36, 1526-1529. (b) Maraval. V.: Laurent, R.: Donnadieu. B.: Mauzac M.: Caminade, A. M.: Majoral. J.-P. J. Am. Chent Soc. 2000. 122. 2499-2511. (c) Mekelburger. H.-B.: Rissanet1. K.: Vögtle. F. Chent Ber: 1993. 126.1161-1169.

4. (a) Kim. C.: Kwon. A. Smbesis 1998. 105-108. (b) Kim. C.: Park. J. Symhesis 1999. 1804-1808.

5. Kim. C. Park, J. J. Organonet. Chem. 2001. 629. 194-200. 